Original Article (short paper)

\title{
Cardiac autonomic responses during and after a single session of aerobic exercise with and without blood flow restriction
}

\author{
Julio Cezar Schamne ${ }^{1}$, Adalberto Ferreira Junior ${ }^{1,2}{ }^{\circledR}$, Amanda Cristiane de Araújo ${ }^{1}$, \\ Adriano Eduardo Lima-Silva ${ }^{3}$, Rômulo Cássio de Moraes Bertuzzi ${ }^{4}$, Nilo Massaru Okuno ${ }^{1}$ \\ ${ }^{1}$ Universidade Estadual de Ponta Grossa, Departamento de Educação Física, Ponta Grossa, PR, Brasil; \\ ${ }^{2}$ Universidade Estadual de Londrina, Departamento de Educação Física, Londrina, PR, Brasil; ${ }^{3}$ Universidade \\ Tecnológica Federal do Paraná, Departamento de Educação Física e Ciências do Esporte, Curitiba, PR, \\ Brasil; ${ }^{4}$ Universidade de Sao Paulo, Escola de Educação Física e Esporte, São Paulo, SP, Brasil
}

\begin{abstract}
Aim: To verify the on and off heart rate (HR) and HR variability (HRV) kinetics during a high-load aerobic exercise and a low-load aerobic exercise, with and without blood flow restriction (BFR). Methods: Fourteen healthy male subjects performed three randomly assigned experimental sessions: 1) 10 minutes walking at $40 \%$ of maximal aerobic speed (MAS) (40LL); 2) 10 minutes walking at 40\% of MAS with BFR (40LL+BFR); and 3) 10 minutes running at $70 \%$ of MAS $(70 \mathrm{HL})$. The HR and HRV measurements were taken at rest, during exercise and the recovery period after constant load sessions. Results: The HR on- and off- kinetics, and HRV on-kinetics were faster in 40LL than in 40LL +BFR and 70HL $(p<0.05)$. The time constant $\left(\tau_{\text {on }}\right)$ of HR on-kinetics was faster in 40LL+BFR than in 70HL $(23.4 \pm 9.5 \mathrm{~s} v s 42 \pm 9.5 \mathrm{~s}$, respectively, $\mathrm{p}<0.01)$, and was accompanied by faster HRV on-kinetics $(12.4 \pm 9.6 \mathrm{~s} v s 20.3 \pm 13.7 \mathrm{~s}$, respectively, $\mathrm{p}<0.01)$. Although HR off-kinetics was not different between 40LL+BFR and 70HL, the recovery of time and frequency HRV indices were delayed in 70HL when compared to 40LL and 40LL+BFR $(p<0.05)$. Conclusions: These findings indicate that 40LL promoted faster cardiac adjustments compared to 40LL+BFR and 70HL sessions. Additionally, 40LL+BFR promoted faster cardiac adjustment and better HRV recovery compared to the 70HL session.
\end{abstract}

Keywords: vascular occlusion, acute responses, heart rate, heart rate variability.

\section{Introduction}

Monitoring heart rate (HR) and HR variability (HRV) permit verification of the autonomic nervous system response (ANS) to different conditions, such as rest, exercise, and post-exercise recovery ${ }^{1}$. The changes in cardiac autonomic modulation from rest to exercise (on-transient) and from exercise to rest, during post-exercise recovery (off-transient), are important to maintaining homeostasis ${ }^{2,3}$. The abrupt increase in HR at the onset of exercise can be attributed to parasympathetic activity withdrawal, followed by an increase in sympathetic activity ${ }^{4}$. During passive recovery, parasympathetic reactivation occurs, which is followed by progressive sympathetic withdrawal ${ }^{5}$.

Mathematical models such as HR on- and off-kinetics are used to verify cardiac autonomic responses during and after exercise $^{1,4}$. In addition, it has been suggested that slow HRV on-kinetics, as evidenced by the root-mean-square difference in successive normal RR intervals (RMSSD), could be related to lower vagal withdrawal at the beginning of the exercise, and reflects an inadequate autonomic response to exercise ${ }^{1}$. Thus, vagal withdrawal and an increase in sympathetic activity influence the HR and HRV on-kinetics. On the other hand,
HR off-kinetics has been related to cardiac parasympathetic reactivation after exercise ${ }^{1,4 .}$ Moreover, some indices of HRV recovery (e.g. RMSSD $60 \mathrm{~s}$ ) can be used to assess parasympathetic reactivation following exercise ${ }^{6}$.

Cardiac adjustment at the beginning of exercise guarantees adequates oxygen supply to active muscles ${ }^{7,8}$. Abnormally slower HR on-kinetics might promote inadequate blood supply to working muscles, which could increase the rating of perceived exertion (RPE) and consequently lead to premature fatigue $^{2}$. Similarly, HR off-kinetics has been related to cardiac parasympathetic reactivation after exercise; therefore, negative changes in ANS (e.g. metabolic syndrome, aging process) cause slower HR off-kinetics ${ }^{2,3}$. It has also been proposed that a slower return of cardiovascular parameters to resting values after exercise is related to increased cardiovascular risk and mortality ${ }^{4}$.

An interesting and new approach consists of combining aerobic training with blood flow restriction (BFR). It has been reported that combining BFR with aerobic exercise improves $\mathrm{VO}_{2 \max }{ }^{9,10}$ and promotes similar gains in muscle strength and hypertrophy to high-load exercise in young healthy men ${ }^{11}$. Furthermore, several studies compared the acute responses of HR and RPE during resistance ${ }^{12,13}$ and aerobic exercise ${ }^{14}$ and 
verified equal or lower hemodynamic responses during or after exercise when performed with BFR compared to high-load exercise without BFR. Additionally, in the study of Ferreira, et al. ${ }^{15}$, lower hemodynamic responses and improvement in time and frequency domain indices of HRV were verified during recovery from low-load aerobic exercise with BFR compared to a high-load session in elderly participants. However, these authors did not investigate the HR and HRV responses during exercise. Thus, it is not clear whether aerobic exercise with BFR could affect cardiovascular responses during and after exercise. This knowledge is important to better understand the cardiovascular adjustments during on and off transitions in aerobic exercise combined with BFR, to demonstrate whether this protocol could promote lower cardiovascular stress than high-load aerobic exercise, decreasing the risk of cardiovascular events ${ }^{16}$.

Therefore, this study aimed to investigate the HR on- and off-kinetics, HRV on-kinetics, and HRV recovery during high-load aerobic exercise and low-load aerobic exercise, with and without BFR.

\section{Methods}

\section{Participants}

Fourteen male subjects, physically active, took part in this study (Table 1). The subjects were considered physically active according to the American College of Sports Medicine ${ }^{17}$. The inclusion criteria were: (a) not having any previous experience in blood flow restriction training, (b) the absence of any kind of cardiovascular or metabolic disease, (c) no articular or bone injury, and (d) not using any medication that could influence cardiovascular response. All participants were aware of the procedures and risks of the experimental protocol and signed an informed consent. The study was approved by the local Ethics Committee (number protocol approved 2.130.924/2017) and performed in accordance with the ethical standards.

Table 1. Sample characteristics $(n=14)$

\begin{tabular}{lc}
\hline Age (years) & $24.1 \pm 4.8$ \\
Height $(\mathrm{cm})$ & $175.2 \pm 6.5$ \\
Weight $(\mathrm{kg})$ & $76.8 \pm 13.4$ \\
Body mass index $\left(\mathrm{kg} / \mathrm{m}^{2}\right)$ & $25.1 \pm 3.8$ \\
Heart rate $(\mathrm{bpm})$ & $70.2 \pm 13.1$ \\
Systolic blood pressure $(\mathrm{mmHg})$ & $115.8 \pm 6.4$ \\
Diastolic blood pressure $(\mathrm{mmHg})$ & $72.1 \pm 6.7$ \\
Maximal aerobic speed $(\mathrm{km} / \mathrm{h})$ & $16.9 \pm 1.9$ \\
\hline
\end{tabular}

\section{Experimental Design}

Data collection was performed on four non-consecutive days. On the first day, anthropometric and resting HRV measurements were taken. Moreover, the participants underwent a maximal incremental test on a treadmill (Inbramed Millennium, model ATL, Porto Alegre) to determine maximal aerobic speed (MAS). The test started at $4 \mathrm{~km} / \mathrm{h}$, with increments of $1 \mathrm{~km} / \mathrm{h}$ every
1 min and a constant slope of $1 \%$, until voluntary exhaustion. The MAS was defined as the final completed stage achieved by subjects in the incremental test.

On the remaining visits, the participants returned to the laboratory on different days to perform 10 minutes walking at $40 \%$ of MAS with and without BFR (40LL +BFR and 40LL, respectively) and 10 minutes running at $70 \%$ of MAS without BFR (70HL). All the subjects performed the three randomly assigned experimental sessions (40LL, 40LL+BFR and 70HL). The subjects were instructed not to ingest alcohol or caffeinated beverages and not to perform vigorous physical activity $24 \mathrm{~h}$ prior to any visit to the laboratory. All the sessions were conducted in the same period of the day to avoid circadian effects on the main outcomes.

A schematic representation of the experimental design is presented in Figure 1.

\begin{tabular}{|c|c|c|c|c|c|}
\hline First day & & \multicolumn{3}{|c|}{ Experimental sessions } & Recovery \\
\hline \multirow{2}{*}{$\begin{array}{l}\text { Anthropometric } \\
\text { HRV } \\
\text { Incremental test }\end{array}$} & $48-72 \mathrm{~h}$ & $40 \mathrm{LL}$ & 40LL+BFR & 70HL: & HR and HRV assessment \\
\hline & \multicolumn{5}{|c|}{ Randomly Assigned } \\
\hline
\end{tabular}

Experimental sessions

40LL- $40 \%$ of maximal aerobic speed without BFR 40LL+BFR- $40 \%$ of maximal aerobic speed with BFR 70HL- $70 \%$ of maximal aerobic speed without BFR

The interval between sessions was $48-72$ hours

Figure 1. Schematic representation of the experimental design.

\section{Experimental sessions}

The subjects underwent constant load tests on the same treadmill with a slope of $1 \%$. The RR intervals were continuously recorded during $3 \mathrm{~min}$ standing at rest, during the entire exercise and during 10 min of recovery standing on the treadmill using a portable heart rate monitor (Polar V800, Kempele, Finland) with a belt fastened around the chest. In the 40LL+BFR session, a standard nylon blood pressure cuff (18 cm (width) $80 \mathrm{~cm}$ (length)) was attached in the inguinal fold region of the participant's thigh only during exercise. The pressure adopted was $100 \mathrm{mmHg}$, based on Laurentino, et al. ${ }^{18}$, this pressure is approximately $80 \%$ of the pressure that causes total restriction of blood flow to the tibial artery.

The rating of perceived exertion (RPE) was measured at the end of each session using a 15-point Borg scale ${ }^{19}$.

\section{$H R$ and HRV kinetics measurements}

The HR (on- and off-transients) and HRV (on-transient) kinetics were calculated through the software Origin 8. For the assessment of the HR on-transient, we used the method described by D'Agosto, et al. ${ }^{1}$ and Javorka, Zila, Balhárek, Javorka ${ }^{4}$. Initially, HR values were calculated at a given time from $R R$ intervals ( $H R=60 / R R$ intervals). Next, the first $300 \mathrm{~s}$ of the exercise HR curve was fitted by a first-order exponential 
function, described by the equation: $\mathrm{HR}_{(\mathrm{t})}=\mathrm{HR}_{0}+\mathrm{A}_{\text {on }}$ $\left\{1-\exp \left[-\left(\mathrm{t}-\mathrm{t}_{o n}\right) / \tau_{o n}\right]\right\}$. Where $\mathrm{HR}(\mathrm{t})$ represents the HR at a given time of the exercise; $\mathrm{HR}_{0}$ corresponding to the asymptotic portion of the curve; $\mathrm{A}_{\text {on }}$ is the amplitude of the HR response; $t_{o n}$ is the time of the exercise onset; and $\tau_{o n}$ is the time constant of the HR response.

For the HR off-transient, we utilized the method described by Javorka, Zila, Balhárek, Javorka ${ }^{4}$. The first 300 seconds of the HR values of the recovery onset period were considered to calculate the fitting curve through the equation: $\mathrm{HR}_{(\mathrm{t})}=\mathrm{HR}_{\min }+\mathrm{A}_{\text {off }} \mathrm{x} \exp \left[-\left(\mathrm{t}-\mathrm{t}_{\text {off }}\right) / \tau_{\text {off }}\right]$. Where $\mathrm{HR}_{(\mathrm{t})}$ represents the $H R$ at a given time of the recovery; $H_{\text {min }}$ is the hypothetical HR level to which the computed exponential curve approaches asymptotically; A off is the amplitude of the HR response; toff is the time of the recovery onset; and $\tau_{\text {off }}$ is the time constant of the HR response.

We used the method described by D'Agosto, et al. ${ }^{1}$ for the HRV on-transient. Initially, the RMSSD was calculated in consecutive and overlapped $15 \mathrm{~s}$ segments throughout the $300 \mathrm{~s}$ of exercise using the software SinusCor. The calculation was performed with a $1 \mathrm{~s}$ step between segments, resulting in 286 RMSSD values that were fitted by the equation: RMSSD15(t) $=$ RMSSD $150+\mathrm{A}_{\text {on }} \mathrm{x} \exp$ $\left[-\mathrm{t} / \tau_{\text {on }}\right]$. Where RMSSD15(t) is the RMSSD value during a given time of the exercise; RMSSD150 is the RMSSD value for the asymptotic portion of the curve; $A_{o n}$ is the amplitude of the RMSSD response; $t$ is the independent variable (time); and ton is the time constant for the RMSSD response.

\section{Heart rate variability and blood pressure assessment during recovery}

On the first day, all participants remained seated in a chair during 10 minutes for HRV recording and blood pressure assessment through an automated blood pressure cuff (Omron HEM-631 INT, Digital BP monitor, Omron Healthcare, The Netherlands). Moreover, the HRV was collected during the 10-min recovery after each experimental session, in a standing position. In both cases, the final 5 min was used to analyze the time- and frequency-domains of HRV. The time-domain variables calculated were: RRi (RR intervals), SDNN (standard deviation of consecutive RR intervals), and RMSSD (root mean square successive differences between adjacent normal RRi). The frequency-domain parameters were obtained through fast Fourier transform (FFT), using Kubios HRV software (version 3.0). High-frequency ( $\mathrm{HF}, 0.15-0.50 \mathrm{~Hz}$ ) and low-frequency (LF, 0.04-0.15 Hz) components were expressed in both absolute and normalized units and the LF/HF ratio was calculated ${ }^{20}$. The time-varying vagal-related index (RMSSD on subsequent $30 \mathrm{~s}$ non-overlapped segments - RMSSD30s) was calculated only during the entire $10 \mathrm{~min}$ of the recovery period. According to Peçanha, Bartels, Brito, Paula-Ribeiro, Oliveira, Goldberger ${ }^{6}$, this method is reliable to verify the parasympathetic reactivation after exercise. Thus, it was not considered RMSSD30s in the rest period to compare with the constant load sessions.

The blood pressure was also measured at $5^{\text {th }}$ and $10^{\text {th }}$ minute of recovery after constant load tests.

\section{Statistical analysis}

The data are presented as mean \pm SD. The Gaussian distribution was observed by the Shapiro-Wilk test. Depending on the distribution of the data, repeated-measures ANOVA was used followed by the Bonferroni post-hoc test or Friedman test and then by the Wilcoxon test to compare the HR and HRV kinetics, HRV indices, blood pressure values and ratings of perceived exertion. All analyses were performed using the software IBM SPSS Statistics (version 21). The significance level was set at $\mathrm{p}<0.05$.

\section{Results}

The average heart rate during exercise in $70 \mathrm{HL}(162 \pm 10 \mathrm{bpm})$ was higher than in 40LL+BFR $(123 \pm 12 \mathrm{bpm}$; $\mathrm{p}<0.001)$ and 40LL $(108 \pm 10 \mathrm{bpm} ; \mathrm{p}<0.001)$. Furthermore, the heart rate in $40 \mathrm{LL}+\mathrm{BFR}$ was significantly higher than in 40LL $(\mathrm{p}<0.01)$.

The HR and HRV kinetics parameters are summarized in Table 2. In HR on-transient kinetics, it was observed that 70HL showed higher values of time constant $\left(\tau_{o n}\right)$ and amplitude $\left(\mathrm{A}_{o n}\right)$ when compared to 40LL and 40LL+BFR $(\mathrm{p}<0.01)$. In addition, $\tau_{\text {on }}$ was lower in $40 \mathrm{LL}$ when compared to $40 \mathrm{LL}+\mathrm{BFR}(\mathrm{p}<0.05)$.

Table 2. Mean and SD of heart rate on- and off-transient kinetics and heart rate variability on-transient kinetics during the constant load aerobic exercise for each experimental condition.

\begin{tabular}{lccc}
\hline & 40LL & 40LL+BFR & 70HL \\
\hline HR kinetics & & & \\
On-transient & & & \\
$\tau_{\text {on }}(\mathrm{s})$ & $14.8 \pm 9.6^{*}$ & $23.4 \pm 9.5^{*} \#$ & $42 \pm 9.5$ \\
A on $(\mathrm{bpm})$ & $26.8 \pm 6.4^{*}$ & $39.1 \pm 14.9^{*}$ & $76.1 \pm 8$ \\
Off-transient & & & \\
$\tau_{\text {off }}(\mathrm{s})$ & $31.9 \pm 21.1^{*}$ & $51.1 \pm 19.3 \#$ & $71.7 \pm 25.3$ \\
Aoff $(\mathrm{bpm})$ & $30.6 \pm 6.4^{*}$ & $39 \pm 10^{*} \#$ & $67.2 \pm 10$ \\
HRV kinetics & & & \\
$\tau_{\text {on }}(\mathrm{s})$ & $5.5 \pm 5.1^{*}$ & $12.4 \pm 9.6^{*} \#$ & $20.3 \pm 13.7$ \\
A on $(\mathrm{bpm})$ & $11.2 \pm 10.9$ & $16.5 \pm 15.5$ & $8.8 \pm 13.1$ \\
\hline
\end{tabular}

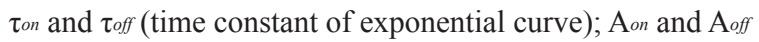
(amplitude of exponential curve). 40LL: 10 minutes of aerobic exercise at $40 \%$ of maximal aerobic speed; $40 \mathrm{LL}+\mathrm{BFR}: 10$ minutes of aerobic exercise at $40 \%$ of maximal aerobic speed with blood flow restriction; 70HL: 10 minutes of aerobic exercise at $70 \%$ of maximal aerobic speed.

* Significantly different from 70HL $(\mathrm{p}<0.01)$

\# Significantly different from 40LL $(\mathrm{p}<0.05)$

In the transition from exercise to recovery, $\tau_{\text {off }}$ was lower in 40LL when compared to 40LL+BFR and 70HL $(\mathrm{p}<0.05)$. Moreover, there was no significant difference between 40LL+BFR and 70HL $(\mathrm{p}>0.05)$. In relation to the $\mathrm{A}_{\text {off }}$ parameter, the 40LL presented significantly lower Aoff than 40LL+BFR and 70HL $(\mathrm{p}<0.05)$ and the 70HL was higher than 40LL+BFR $(\mathrm{p}<0.01)$ (Table 2).

For HRV on-transient, $\tau_{\text {on }}$ was higher in $70 \mathrm{HL}$ compared to 40LL and 40LL +BFR ( $<<0.01)$, and higher in 40LL + BFR 
compared to 40LL $(p<0.05)$. There were no significant differences for $\mathrm{A}$ on in HRV on-transient when comparing the experimental sessions $(\mathrm{p}>0.05)$ (Table 2$)$.

The time- and frequency-domain indices of HRV are summarized in Table 3. The RRi, SDNN, and RMSSD were all reduced post-exercise in all experimental conditions when compared with rest $(p<0.05)$. In addition, RRi, SDNN, and RMSSD were all lower in 70HL compared to 40LL and 40LL +BFR $(\mathrm{p}<0.01)$ and the RRi index was significantly lower in 40LL+BFR compared to 40LL $(\mathrm{p}<0.05)$.

Table 3. Mean and SD of time and frequency domain indices of heart rate variability at rest and in the $5-10^{\text {th }}$ min of recovery after constant load aerobic exercise for each experimental condition.

\begin{tabular}{lcccc}
\hline & Rest & 40LL & 40LL+BFR & 70HL \\
\hline RRi (ms) & $915.4 \pm 153.5$ & $726.6 \pm 85.9^{* \#}$ & $680.6 \pm 86^{* \# \dagger}$ & $583.8 \pm 42.6^{*}$ \\
SDNN (ms) & $54.7 \pm 15.2$ & $40.6 \pm 11.4^{* \#}$ & $38.2 \pm 14.6^{* \#}$ & $21.3 \pm 9.3^{*}$ \\
RMSSD (ms) & $45.7 \pm 15.1$ & $21.8 \pm 8^{* \#}$ & $21.1 \pm 9.5^{* \#}$ & $11.1 \pm 5.8^{*}$ \\
LF $\left(\mathrm{ms}^{2}\right)$ & $2206.5 \pm 1550.5$ & $1650.7 \pm 1008.8^{\#}$ & $1464.5 \pm 1202.8^{* \#}$ & $440.7 \pm 292.5^{*}$ \\
HF $\left(\mathrm{ms}^{2}\right)$ & $856.9 \pm 522.7$ & $226.9 \pm 175.5^{* \#}$ & $248.3 \pm 237.7^{* \#}$ & $57.9 \pm 48.5^{*}$ \\
LF (nu) & $70.5 \pm 14.7$ & $87.7 \pm 7.8^{*}$ & $84.1 \pm 13.1^{*}$ & $88.7 \pm 6.2^{*}$ \\
HF (nu) & $29.5 \pm 14.7$ & $12.6 \pm 7.8^{*}$ & $12.7 \pm 8.7^{*}$ & $11.3 \pm 6.1^{*}$ \\
LF/HF & $3.1 \pm 1.2$ & $10 \pm 6.1^{*}$ & $7.6 \pm 4.8^{*}$ & $11.6 \pm 8.9^{*}$ \\
\hline
\end{tabular}

40LL: 10 minutes of aerobic exercise at 40\% of maximal aerobic speed; 40LL+BFR: 10 minutes of aerobic exercise at $40 \%$ of maximal aerobic speed with blood flow restriction; 70HL: 10 minutes of aerobic exercise at $70 \%$ of maximal aerobic speed.

* Significantly different from rest $(\mathrm{p}<0.05)$

\# Significantly different from 70HL $(\mathrm{p}<0.05)$

$\uparrow$ Significantly different from 40LL $(\mathrm{p}<0.05)$

For the frequency domain analysis, LFnu and LF/HF were increased, while $\mathrm{HF}\left(\mathrm{ms}^{2}\right)$ and HFnu reduced after all exercises, compared with rest $(\mathrm{p}<0.05)$. The LF $\left(\mathrm{ms}^{2}\right)$ was also decreased in 40LL+BFR and 70HL compared with rest $(\mathrm{p}<0.05)$. Moreover, $\mathrm{LF}\left(\mathrm{ms}^{2}\right)$ and $\mathrm{HF}\left(\mathrm{ms}^{2}\right)$ indices were higher in 40LL and 40LL+BFR than in 70HL $(p<0.01)$. There were no significant differences between 40LL and 40LL+BFR for any frequency-domain parameters $(\mathrm{p}>0.05)$. The post-exercise RMSSD30s is shown in Figure 2. The RMSSD30s was reduced during the entire period in 70HL compared to 40LL and 40LL +BFR ( $p<0.05)$. For the first $90 \mathrm{~s}$, the RMSSD30s was also reduced in 40LL +BFR compared to 40LL $(p<0.05)$. Additionally, it was not observed differences for both systolic and diastolic blood pressure measured at $5^{\text {th }}$ and $10^{\text {th }}$ minute of recovery among the constant load sessions $(p>0.05)$ (Table 4$)$.

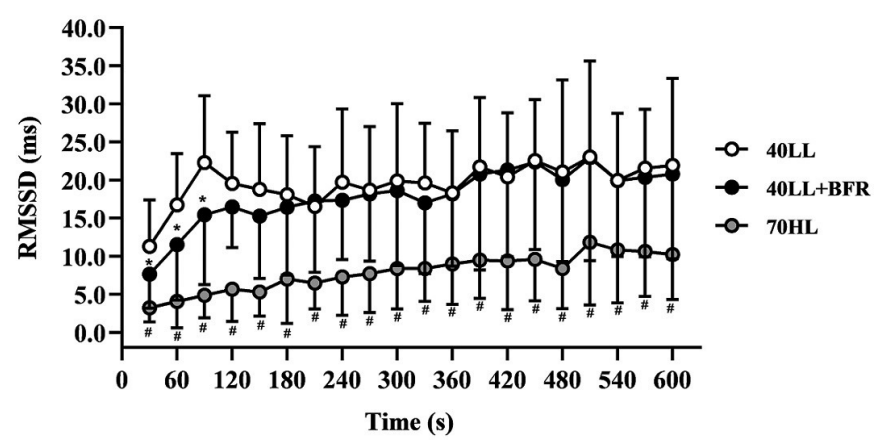

Figure 2. Mean and SD of root-mean-square of successive differences in the RR intervals measured in successive 30 s segments (RMSSD30s) during the $10 \mathrm{~min}$ recovery period after the constant load exercise for each experimental condition.

* Significantly different from 40LL $(\mathrm{p}<0.05)$

\# Significantly different from 40LL and 40LL+BFR $(p<0.01)$
Table 4. Mean and SD of systolic and diastolic blood pressure at 5th and 10th min of recovery after constant load aerobic exercise for each experimental condition.

\begin{tabular}{cccc}
\hline & 40LL & 40LL+BFR & 70HL \\
\hline SBP $(\mathrm{mmHg})$ & & & \\
$5^{\text {th }}$ minute & $113.5 \pm 8.49$ & $113.4 \pm 4.9$ & $111.1 \pm 8.7$ \\
$10^{\text {th }}$ minute & $114.1 \pm 12.8$ & $111.9 \pm 5.9$ & $107.8 \pm 6.4$ \\
DBP $(\mathrm{mmHg})$ & & & \\
$5^{\text {th }}$ minute & $69.9 \pm 9.6$ & $71.5 \pm 7.1$ & $68.1 \pm 8.1$ \\
$10^{\text {th }}$ minute & $72.2 \pm 9.5$ & $71.6 \pm 4.4$ & $70.1 \pm 5.6$ \\
\hline
\end{tabular}

SBP: Systolic blood pressure; DBP: Diastolic blood pressure. 40LL: 10 minutes of aerobic exercise at $40 \%$ of maximal aerobic speed; 40LL+BFR: 10 minutes of aerobic exercise at $40 \%$ of maximal aerobic speed with blood flow restriction; 70HL: 10 minutes of aerobic exercise at $70 \%$ of maximal aerobic speed.

As expected, the RPE in 40LL was significantly lower $(\mathrm{p}<0.01)$ than 40LL+BFR and 70HL. Furthermore, no differences were observed between 40LL+BFR and 70HL $(p>0.05)$ (Table 5).

Table 5. Ratings of perceived exertion (RPE) at the constant load sessions.

\begin{tabular}{ccc}
\hline 40LL & 40LL+BFR & 70HL \\
\hline $9.6 \pm 1.4^{*}$ & $12.7 \pm 2.3$ & $14.7 \pm 2.4$ \\
\hline
\end{tabular}

* Significantly different from 40LL+BFR and 70HL $(p<0.01)$

\section{Discussion}

The main results of the present study revealed that (1) both HR on- and off-transient kinetics are delayed with BFR, (2) only HR off-transient kinetics were similar to that found during 
a high-load exercise, and (3) the post-exercise vagal control for the first 90s of the recovery, as represented by the RMSSD, seems also to be impaired with BFR, but does not match with post high-load exercise values.

To our knowledge, this is the first study to verify the HR and HRV on-transient kinetics in low-load aerobic exercise with BFR and compare this response with high-load aerobic exercise. Slower and faster cardiovascular adjustments were demonstrated for low-load exercise with BFR when compared to low-load exercise without BFR and high-load exercise, respectively. This is in accordance with previous studies that investigated cardiovascular adjustments in different intensities of aerobic exercise $\mathrm{e}^{21,22}$. From rest to exercise transition, the skeletal muscle contractions promote a parasympathetic withdrawal in the first few seconds via muscle mechanoreflex ${ }^{23}$ and gradual sympathetic activation mediated principally via muscle metaboreflex ${ }^{24}$. The higher physiological disturbance during high exercise intensities may contribute to the delayed achievement of a steady state in $\mathrm{HR}^{25}$, which might explain the higher $\tau_{\text {on }}$ value of HR and HRV kinetics in 70HL. Additionally, it is believed that exercise at low-load with BFR promotes larger muscle metabolite [e.g. lactate, $\mathrm{H}^{+}$] accumulation provided by tissue hypoxia and its inadequate removal caused by limited venous return, and this condition activates the muscle metaboreflex, which increases HR at the onset of exercise and during low-load steady-state exercise ${ }^{26,27}$. This might explain the higher HR amplitude and slower adjustments of HR and HRV on-transient kinetics (higher $\tau$ value) in $40 \mathrm{LL}+\mathrm{BFR}$, compared to without BFR.

During exercise recovery there is faster parasympathetic reactivation, leading to $\mathrm{HR}$ deceleration ${ }^{28}$; previous studies have related the HR recovery to exercise intensity ${ }^{29,30}$. For example, Mann, Webster, Lamberts, Lambert ${ }^{30}$, reported slower HR recovery after exercise performed at $80 \%$ compared to $60 \%$ of $\mathrm{VO}_{2 \max }$. Despite the parasympathetic reactivation, the exerciseinduced an increase in sympathetic nervous system activation at higher intensities, promoting slower sympathetic withdrawal in post-exercise recovery ${ }^{28}$. Interestingly, no differences were found between 40LL+BFR and 70HL for $\tau_{\text {off }}$, but the Aoff was lower in 40LL+BFR. On the other hand, the lower A off during the 40LL+BFR suggests lower hemodynamic stress when compared to $70 \mathrm{HL}^{15}$.

The present study demonstrated similar values in time and frequency domain indices between 40LL and 40LL+BFR analyzed in the $5-10^{\text {th }}$ min of recovery. However, for the first $90 \mathrm{~s}$ of the recovery, the RMSSD was higher in 40LL+BFR than in 40LL but did not match values for $70 \mathrm{HL}$. Few studies have investigated HRV recovery after a BFR exercise. Ferreira, et al. ${ }^{15}$ found similar HRV time- and frequencydomain indices in 20 and 30 minutes of recovery after three sessions of aerobic exercise (low-load, low-load with BFR, and high-load). However, delayed HRV recovery was observed in the study of Ferreira, et al. ${ }^{15}$ compared with the present study, which may be explained due to some methodological differences such as the time of exercise session (20 min) and age of participants (older adults). In another study, Okuno,
Pedro, Leicht, Ramos, Nakamura ${ }^{31}$ also demonstrated different magnitudes of HRV recovery in resistance exercise sessions with and without BFR. The higher demand for active muscles in higher intensity exercises may induce higher metabolite accumulation during exercise ${ }^{32}$ and higher $H R$ values at the cessation of exercise, promoting slower vagal tone recovery and sympathetic withdrawal ${ }^{33}$. These factors could explain the slower HRV recovery in 70HL than 40LL and 40LL+BFR. Additionally, the cardiac autonomic changes promoted by BFR at $40 \%$ of MAS persisted only in the two minutes of recovery, as after this period no significant differences were observed between 40LL and 40LL+BFR. The discrepancies observed between time- and frequency-domain indices in the present study probably occurred due to the absence of controlled respiratory pattern. A reliable spectral analysis for frequency-domain indices of HRV requires a controlled breathing rate, while time domain indices (e.g. SD1, RMSSD) is not affected significantly ${ }^{34}$.

The RPE was larger in 40LL+BFR than in 40LL but was not different from $70 \mathrm{HL}$. Similar results were obtained when comparing high-load exercise with low-load exercise with $\mathrm{BFR}^{14}$. However, in the study of May, Brandner, Warmington ${ }^{14}$ lower RPE was observed when the exercise was performed with BFR compared to a high-load session., The authors utilized an intermittent protocol (4 sets of 2 minutes) and lower intensities of walking $(4 \mathrm{~km} / \mathrm{h})$. These discrepancies in methodology could explain the differences in RPE.

Additionally, it has been suggested that BFR during exercise causes muscle pain and discomfort ${ }^{35,36}$, which could reflect in higher RPE ${ }^{13}$. Therefore, the combination of a greater BFR and higher exercise intensity might have contributed to the higher RPE values in 40LL+BFR when compared to 40LL.

The main limitations of the present study were healthy young subjects recruited, which must not represent the safety of walking with BFR in other populations (e.g. elderly and subjects with cardiovascular disease); not measuring other hemodynamic parameters, which could help us to better understand and explain the responses of aerobic exercise with BFR; despite the use of the same time frame during HRV analysis, the evaluator was not blinded, knowing the protocol that each subject performed; and the choice of using the same absolute pressure for all participants. Notwithstanding, the use of absolute cuff pressure is easier for practical application in dailylife, and this study helped to increase understanding of cardiac autonomic response in aerobic exercise with BFR.

\section{Conclusion}

The present data suggest that 40LL promoted faster cardiovascular adjustment (on-and off-transient) when compared to $40 \mathrm{LL}+\mathrm{BFR}$ and $70 \mathrm{HL}$ sessions. In addition, the 40LL+BFR promoted faster cardiac adjustment and better HRV recovery compared to the 70HL session without post-exercise systolic and diastolic blood pressure difference among the sessions. These results indicate that walking with BRF promotes lower cardiovascular stress than traditional high-load aerobic exercise 
and could be an alternative method for individuals who are unable to perform high-load exercises.

\section{References}

1. D’Agosto T, Peçanha T, Bartels R, Moreira DN, Silva LP, Nóbrega ACL, et al. Cardiac autonomic responses at onset of exercise: effects of aerobic fitness. Int J Sports Med. 2014;35(10):879-885.

2. Silva LRBE, Zamunér AR, Gentil P, Alves FM, Leal AGF, Soares V, et al. Cardiac autonomic modulation and the kinetics of heart rate responses in the on- and off-transient during exercise in women with metabolic syndrome. Front Physiol. 2017;8:1-9.

3. Simões RP, Bonjorno-Jr JC, Beltrame T, Catai AM, Arena R, Borghi-Silva A. Slower heart rate and oxygen consumption kinetic responses in the on- and off-transient during a discontinuous incremental exercise: effects of aging. Braz J Phys Ther. 2013;17(1):69-76.

4. Javorka M, Zila I, Balhárek T, Javorka K. On- and off-responses of heart rate to exercise - relations to heart rate variability. Clin Physiol Funct Imaging. 2003;23(1):1-8.

5. Imai K, Sato H, Hori M, Kusuoka H, Ozaki H, Yokoyama H, et al. Vagally mediated heart rate recovery after exercise is accelerated in athletes but blunted in patients with chronic heart failure. J Am Coll Cardiol. 1994;24(6):1529-1535.

6. Peçanha T, Bartels R, Brito LC, Paula-Ribeiro M, Oliveira RS, Goldberger JJ. Methods of assessment of the post-exercise cardiac autonomic recovery: A methodological review. Int J Cardiol. 2017;15(227)795-802.

7. Amann M, Calbet JAL. Convective oxygen transport and fatigue. J Appl Physiol. 2008;104(3):861-870.

8. Rowell LB, O'Leary DS. Reflex control of the circulation during exercise: chemoreflexes and mechanoreflexes. J Appl Physiol. 1990;69(2):407-418.

9. Abe T, Sakamaki M, Fujita, S Ozaki H, Sugaya M, Sato Y, et al. Effects of low-intensity walk training with restricted leg blood flow on muscle strength and aerobic capacity in older adults. J Geriatr Phys Ther. 2010;33(1):34-40.

10. Park S, Kim JK, Choi HM, Kim HG, Beekley MD, Nho H. Increase in maximal oxygen uptake following 2-week walk training with blood flow occlusion in athletes. Eur J Appl Physiol. 2010;109(4):591-600.

11. Kim D, Singh H, Loenneke JP, Thiebaud RS, Fahs CA, Rossow LM, Young K, Seo DI, Bemben DA, Bemben MG. Comparative effects of vigorous-intensity and low-intensity blood flow restricted cycle training and detraining on muscle mass, strength, and aerobic capacity. J Strength Cond Res. 2016;30(5):1453-1461.

12. Araújo AC, Ferreira Junior A, De-Oliveira SK, Schamne JC, Okuno NM. Physiological and rating of perceived exertion responses to resistance training sessions with and without vascular occlusion. Isokinet Exerc Sci. 2017;25(2):91-96.716.

13. Poton R, Polito MD. Hemodynamic response to resistance exercise with and without blood flow restriction in healthy subjects. Clin Physiol Funct Imaging. 2014;36(3):231-236.

14. May AK, Brandner CR, Warmington SA. Hemodynamic responses are reduced with aerobic compared with resistance blood flow restriction exercise. Physiol Rep. 2017;5(3):1-10.
15. Ferreira MLV, Sardeli AV, De-Souza GV, Bonganha V, Santos LDC, Castro A, et al. Cardiac autonomic and haemodynamic recovery after a single session of aerobic exercise with and without blood flow restriction in older adults. J Sports Sci 2017;35(24):2412-2420.

16. Thompson PD, Franklin BA, Balady GJ, Blair SN, Corrado D, Estes NA, Fulton JE, Gordon NF, Haskell WL, Link MS, Maron BJ, Mittleman MA, Pelliccia A, Wenger NK, Willich SN and Costa F. Exercise and acute cardiovascular events placing the risks into perspective: a scientific statement from the American Heart Association Council on Nutrition, Physical Activity, and Metabolism and the Council on Clinical Cardiology. Circulation. 2007;115(17):2358-2368.

17. American College of Sports Medicine position stand (ACSM). The Recommended Quantity and Quality of Exercise for Developing and Maintaining Cardiorespiratory and Muscular Fitness, and Flexibility in Healthy Adults. Med Sci Sports Exerc. 2011;43(7):1334-1359.

18. Laurentino G, Ugrinowitsch C, Aihara AY, Fernandes AR, Parcell AC, Ricard M, et al. Effects of strength training and vascular occlusion. Int J Sports Med. 2008;29(8):664-667.

19. Borg, GA. Psychophysical bases of perceived exertion. Med Sci Sports Exerc. 1982;14(5):377-381.

20. Ferreira Junior A, Schamne JC, Franzói De-Moraes SM, Okuno NM. Cardiac autonomic responses and number of repetitions maximum after LED irradiation in the ipsilateral and contralateral lower limb. Lasers Med Sci. 2018;33(2):353-359.

21. Lai N, Martis A, Belfiori A, Tolentino-Silva F, Nasca MM, Strainic J, et al. Gender differences in VO2 and HR kinetics at the onset of moderate and heavy exercise intensity in adolescents. Physiol Rep. 2016;4(8):1-12.

22. Trounson KM, Roberts S, Balloch A, Warmington SA. Light exercise heart rate on-kinetics: a comparison of data fitted with sigmoidal and exponential functions and the impact of fitness and exercise intensity. Physiol Rep. 2017;5(12):1-12.

23. Gladwell VF, Coote JH. Heart rate at the onset of muscle contraction and during passive muscle stretch in humans: a role for mechanoreceptors. J Physiol. 2002;540:1095-1102.

24. Fisher JP. Autonomic control of the heart during exercise in humans: role of skeletal muscle afferents. Exp Physiol. 2013;99(2):300-305.

25. Zakynthinaki M. Modelling Heart Rate Kinetics. PLoS One. 2015;10(4):1-26.

26. Renzi CP, Tanaka H, Sugawara J. Effects of leg blood flow restriction during walking on cardiovascular function. Med Sci Sports Exerc. 2010;42(4):726-732.

27. Rowell LB. Neural control of muscle blood flow: importance during dynamic exercise. Clin Exp Pharmacol Physio. 1997;24(2):117-125.

28. Pierpont GL, Voth EJ. Assessing autonomic function by analysis of heart rate recovery from exercise in healthy subjects. Am J Cardiol. 2004;94(1):64-68.

29. Michael S, Jay O, Graham KS, Davis GM. Higher exercise intensity delays postexercise recovery of impedance-derived cardiac sympathetic activity. Appl Physiol Nutr Metab. 2017;42(8):834-840.

30. Mann TN, Webster C, Lamberts RP, Lambert MI. Effect of exercise intensity on post-exercise oxygen consumption and heart rate recovery. Eur J Appl Physiol. 2014;114(9):1809-1820. 
31. Okuno NM, Pedro RE, Leicht AS, Ramos SP, Nakamura FY. Cardiac autonomic recovery after a single session of resistance training with and without vascular occlusion. J Strength Cond Res. 2014;28(4):1143-1150.

32. Spranger MD, Krishnan AC, Levy PD, O'Leary DS, Smith SA. Blood flow restriction training and the exercise pressor reflex: a call for concern. Am J Physiol Heart Circ Physiol. 2015;309(9): H1440-H1452.

33. Fisher JP, Seifert T, Hartwich D, Young CN, Secher NH, Fadel PJ. Autonomic control of heart rate by metabolically sensitive skeletal muscle afferents in humans. J Physiol. 2010;588(7):1117-1127.

34. Penttila J, Helminen A, Jartti T, Kuusela T, Huikuri HV, Tulppo MP, Coffeng R, Scheinin H. Time domain, geometrical and frequency domain analysis of cardiac vagal outflow: effects of various respiratory patterns. Clin Physiol. 2001;21(3):365-376.

35. Wernbom M, Järrebring R, Andreasson MA, Augustsson J. Acute effects of blood flow restriction on muscle activity and endurance during fatiguing dynamic knee extensions at low load. J Strength Cond Res. 2009;23(8):2389-2395.

36. Yasuda T, Loenneke JP, Ogasawara R, Abe T. Influence of continuous or intermittent blood flow restriction on muscle activation during low-intensity multiple sets of resistance exercise. Acta Physiol Hung. 2013;100(4):419-426.

\section{Corresponding author}

Julio Cezar Schamne

Department of Physical Education, State University of Ponta Grossa (UEPG), Av. General Carlos Cavalcanti, 4748 - Campus Uvaranas, Ponta Grossa, PR, 84030-900, Brazil.

Email: juliocezarschamne@gmail.com

Manuscript received on December 4, 2018

Manuscript accepted on May 10, 2019

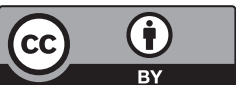

Motriz. The Journal of Physical Education. UNESP. Rio Claro, SP, Brazil - eISSN: 1980-6574 - under a license Creative Commons - Version 4.0 